\title{
DRAwing Conclusions About the Basis of Diarrhea in Inflammatory Bowel Disease
}

\author{
Kim E. Barrett ${ }^{1}$ \\ Published online: 16 January 2020 \\ (c) Springer Science+Business Media, LLC, part of Springer Nature 2020
}

Diarrhea, a characteristic symptom of the inflammatory bowel diseases (IBD) of ulcerative colitis and Crohn's disease, is often the most disabling and distressing disease component. The treatment of these conditions has advanced significantly in recent years, particularly with the advent of a panoply of biologic drugs that can arrest inflammation and trigger mucosal healing. Nevertheless, in some cases, diarrhea can persist in IBD despite evidence that inflammation has abated [1]. In any event, the precise basis of diarrheal symptoms in IBD is only poorly understood, despite the fact that a deeper understanding of diarrheal pathogenesis might inform improved therapy [2]. In general, diarrhea reflects an increased contribution of epithelial secretory mechanisms to luminal fluidity, decreased electrolyte absorption, or some combination of these, while diminished barrier function may also exacerbate underlying transport disturbances [2]. Nonetheless, diarrheal diseases of varying etiologies may exhibit distinct transport dysfunction phenotypes. For example, when considering infectious diarrhea, the diarrhea due to enterotoxigenic pathogens such as Vibrio cholerae is largely driven by excessive chloride secretion, whereas we and others have demonstrated that diarrhea in the setting of infection with invasive pathogens such as non-typhoidal Salmonella spp. more likely involves decreased absorption of $\mathrm{NaCl}$ and sodium ions in the proximal and distal colon, respectively [3].

Murine models have proven important in the quest to unravel the immunopathogenesis of IBD [4]. Disease states resembling IBD can be generated acutely in mice with the administration of chemical irritants into the colon or systemic treatment with antibodies (such as anti-CD3) that activate $\mathrm{T}$ cells; there are also numerous genetic models

Kim E. Barrett

kbarrett@ucsd.edu

1 Department of Medicine, University of California San Diego, Mail Code 0063, 9500 Gilman Drive, La Jolla, CA 92093-0063, USA that are accompanied by epithelial barrier dysfunction, the over-expression of proinflammatory cytokines, or the underexpression of anti-inflammatory cytokines such as IL-10. In general, no single mouse model represents all facets of human IBD, although specific models may more faithfully reflect the immunological phenotype of ulcerative colitis versus Crohn's disease [4]. Nevertheless, the adoptive transfer mouse model of IBD has found favor with many investigators. This is due to its production of disease that occurs at a predictable time point following initial treatment (as also seen in chemical models, such as dextran sodium sulfate [DSS] colitis), but also because, unlike the DSS model, chronic disease results that is more in keeping with human IBD. Yet, the extent to which the diarrheal phenotype that is observed in this model depends on specific alterations in the expression or function of epithelial transport proteins had not been elucidated.

In the current issue of Digestive Diseases and Sciences, Jayawardena and colleagues present important information needed to address the knowledge gaps regarding the basis of diarrhea in the adoptive $\mathrm{T}$ cell transfer model of colitis [5]. Immunodeficient $\mathrm{Rag} 2^{-/-}$mice were administered $\mathrm{CD} 4{ }^{+} \mathrm{CD} 45 \mathrm{RB}^{\text {high }} \mathrm{T}$ cells, reproducibly showing expected increases in colonic proinflammatory cytokines within 4 weeks, without discernable effects on colonic anti-inflammatory cytokines or on ileal cytokines. Moreover, the mice displayed colons that were shortened and contained loose fecal material, compared to the fecal pellets seen in control mice, consistent with a diarrheal phenotype following $\mathrm{T}$ cell transfer. The authors hypothesized that the diarrheal symptoms likely occurring in these mice reflected alterations in the expression of one or more epithelial transporters, subsequently cataloging key transporters by measuring mRNA and protein levels for these, along with studying the localization of one specific transporter by immunofluorescence. In keeping with prior observations in other colitis and infectious diarrhea models in mice, as well as the authors' extensive studies of model cell lines, $\mathrm{T}$ cell transfer colitis 
nearly abolished expression of the chloride/bicarbonate exchanger Down-Regulated in Adenoma (DRA, Slc26a3) in both the proximal and distal colon, without affecting ileal DRA expression (also a prominent site of DRA expression at baseline). DRA typically acts in concert with the sodium/ hydrogen exchanger, NHE3 (Slc9a3) to mediate the electroneutral uptake of $\mathrm{NaCl}$, but interestingly, levels of NHE3 mRNA and protein were unaltered by $\mathrm{T}$ cell transfer in either the colon or ileum. Nevertheless, some other colonic transporters were suppressed in this colitis model, including putative anion transporter 1 (PAT1, Slc26a6) and the alpha subunit of the epithelial sodium channel $(\mathrm{ENaC})$. Of interest, reduced expression and/or function of both DRA and $\mathrm{ENaC}$ was reported in biopsy samples obtained from human patients suffering from IBD [6]. Jayawardena and colleagues also showed that the colitis model was accompanied by reductions in mRNA for two colonic monocarboxylate transporters, MCT1 and SMCT1 (Slc16a1 and Slc5a8, respectively), without any effect on mRNA expression for the anion channel, cystic fibrosis transmembrane conductance regulator (CFTR), in either the colon or the ileum.

To the extent that the findings here are reflective of human IBD in general, and Crohn's disease in particular, the results may point to new strategies aimed at ameliorating diarrheal symptoms in these diseases. Even though NHE3 was not down-regulated, this transporter, coupled with DRA, mediates $\mathrm{NaCl}$ uptake, particularly in the interdigestive period. The loss of DRA alone, therefore, should be sufficient to result in diarrhea, particularly when coupled with a reduction in $\mathrm{ENaC}$ (primarily a salvage pathway for electrogenic sodium reabsorption in the distal colon) as well as an anticipated increase in gut paracellular permeability that could potentiate diarrhea via leak-flux mechanisms [7]. Furthermore, the pattern of electrolyte transport changes seen here has also been observed in infectious diarrheal models, meaning that any therapeutic strategies based on these findings might be more broadly applicable. The authors themselves have previously reported on a number of agents that specifically upregulate the expression of DRA, at least in cell lines, including probiotics, vitamin A metabolites, and lysophosphatidic acid [8-10]. The finding that expression of two monocarboxylate transporters was also suppressed in this model may have further pathophysiological significance, since these molecules transport key short chain fatty acids that are important bioactive signaling molecules and nutrients. Indeed, butyrate itself stimulates DRA expression, suggesting a possible link between the various responses observed [11].

Despite these data that enhance the understanding of the pathogenesis of inflammatory diarrhea, some additional studies might drive the story further. For example, in mice infected with Salmonella typhimurium, while there was no change in CFTR expression at the mRNA or protein level, the channel was apparently mislocalized away from the apical membrane, likely accounting for a reduction in the capacity for crypt chloride secretion in infected tissues [3]. If a similar event occurs in the current model of colitis, it might have implications for host defense, particularly in the sensitive stem cell niche at the crypt base. Overall, information about mRNA, protein, transporter localization, and function is needed to fully understand the pathogenesis of diarrhea as well as other possible contributors to the chronic nature of IBD. Nevertheless, the current study by Jayawardena and colleagues is an excellent contribution to the growing body of knowledge about how diarrhea occurs in the setting of intestinal inflammation, as well as providing pointers as to how it might ultimately be mitigated.

Acknowledgments Studies from the authors' laboratory related to the subject matter of this article have been supported by grants from the National Institutes of Health as well as an unrestricted grant from the Estratest Settlement Fund.

\section{Compliance with Ethical Standards}

Conflict of interest The author has no relevant conflict to disclose.

\section{References}

1. Barros LL, Farias AQ, Rezaie A. Gastrointestinal motility and absorptive disorders in patients with inflammatory bowel diseases: prevalence, diagnosis and treatment. World J Gastroenterol. 2019;25:4414-4426.

2. Camilleri M, Sellin JH, Barrett KE. Pathophysiology, evaluation, and management of chronic watery diarrhea. Gastroenterology. 2017;152:515-532. e512.

3. Marchelletta RR, Gareau MG, McCole DF, et al. Altered expression and localization of ion transporters contribute to diarrhea in mice with Salmonella-induced enteritis. Gastroenterology. 2013; $145: 1358$.

4. Bamias G, Arseneau KO, Cominelli F. Mouse models of inflammatory bowel disease for investigating mucosal immunity in the intestine. Curr Opin Gastroenterol. 2017;33:411-416.

5. Jayawardena D, Tyagi S, Nazmi A, Olivares-Villagómez D, Dudeja PK. Ion transport basis of diarrhea in a mouse model of adoptive T cell transfer colitis. Dig Dis Sci. (Epub ahead of print). https://doi.org/10.1007/s10620-019-05945-4.

6. Anbazhagan AN, Priyamvada S, Alrefai WA, Dudeja PK. Pathophysiology of IBD associated diarrhea. Tissue Barriers. 2018;6:e1463897.

7. Marchiando AM, Graham WV, Turner JR. Epithelial barriers in homeostasis and disease. Annu Rev Pathol. 2010;5:119-144.

8. Priyamvada S, Anbazhagan AN, Kumar A, et al. All-trans retinoic acid counteracts diarrhea and inhibition of downregulated in adenoma expression in gut inflammation. Inflamm Bowel Dis. 2019. https://doi.org/10.1093/ibd/izz249.

9. Kumar A, Anbazhagan AN, Coffing H, et al. Lactobacillus acidophilus counteracts inhibition of NHE3 and DRA expression and alleviates diarrheal phenotype in mice infected with $C i t$ robacter rodentium. Am J Physiol Gastrointest Liver Physiol. 2016;311:G817-G826. 
10. Singla A, Dwivedi A, Saksena S, et al. Mechanisms of lysophosphatidic acid (LPA) mediated stimulation of intestinal apical $\mathrm{Cl}^{-} / \mathrm{OH}^{-}$exchange. Am J Physiol Gastrointest Liver Physiol. 2010;298:G182-G189.

11. Alrefai WA, Wen X, Jiang W, et al. Molecular cloning and promoter analysis of downregulated in adenoma (DRA). Am J Physiol Gastrointest Liver Physiol. 2007;293:G923-G934.
Publisher's Note Springer Nature remains neutral with regard to jurisdictional claims in published maps and institutional affiliations. 